Revue

Revue de l'histoire des religions

de Ihistoire des religions

Özkan BARDAKÇI, François PUGNIÈRE (éd.), La Dernière Croisade. Les Français et la guerre de Candie 1669

Préface de Robert Sauzet, Rennes, Presses Universitaires de Rennes, 2008, 182 p., $24 \mathrm{~cm}$ (« Histoire »), $18 €$.

Chrystel Bernat

\title{
OpenEdition
}

Journals

Édition électronique

URL : http://journals.openedition.org/rhr/7749

DOI : $10.4000 /$ rhr.7749

ISSN : 2105-2573

Éditeur

Armand Colin

Édition imprimée

Date de publication : 1 mars 2011

Pagination : 133-136

ISBN : 978-2200-92685-4

ISSN : 0035-1423

Référence électronique

Chrystel Bernat, «Özkan BARdakçı, François pugnière (éd.), La Dernière Croisade. Les Français et la guerre de Candie 1669 », Revue de l'histoire des religions [En ligne], 1 | 2011, mis en ligne le 30 mai 2011, consulté le 12 octobre 2020. URL : http://journals.openedition.org/rhr/7749 ; DOI : https://doi.org/ 10.4000/rhr.7749

Ce document a été généré automatiquement le 12 octobre 2020

Tous droits réservés 


\section{Özkan BARDAKÇI, François PUGNIÈRE (éd.), La Dernière Croisade. Les Français et la guerre de Candie 1669}

Préface de Robert Sauzet, Rennes, Presses Universitaires de Rennes, 2008, 182 p., $24 \mathrm{~cm}$ (« Histoire »), $18 €$.

\section{Chrystel Bernat}

\section{RÉFÉRENCE}

Özkan BARDAKÇI, François PUGNIÈRE (éd.), La Dernière Croisade. Les Français et la guerre de Candie 1669. Préface de Robert Sauzet, Rennes, Presses Universitaires de Rennes, 2008, 182 p., $24 \mathrm{~cm}$ (« Histoire »), $18 €$.

1 Construit autour de l'édition critique d'un manuscrit inédit, découvert dix ans auparavant par Robert Sauzet, l'ouvrage de Özkan Bardakçi et François Pugnière présente, comme en écho Au Grand Siècle des âmes de leur préfacier (Perrin 2007), une étude de l'expédition française engagée en Crète lors de la cinquième guerre vénétoottomane pour tenter, à l'été 1669 , de sauver la place de Candie menacée, en ce troisième siège, de tomber aux mains des Turcs. L'affaire, de dimension européenne et à forte charge idéologique, est d'emblée saisie dans son ambiguïté fondamentale qui devait faire converger, en deux visées françaises antinomiques, maintien des relations diplomatiques et commerciales avec la Porte et exigence d'alliance chrétienne devant répondre des sollicitations pressantes de Rome et de la République de Venise.

2 Loin des mémoires de guerre fameux, le manuscrit Domenisse dit, en une langue souvent malhabile mais traversée de topoi éloquents et de représentations singulières, l'évolution de l'image du Turc. Tandis qu'il ne pourrait être qu'un prétexte à l'étude plus générale de la guerre, le Mémoire des choses les plus remarquables qui se sont faites au voyage de Candie sert de pivot au questionnement du renversement paradigmatique de la perception de l'Infidèle, aux prises avec le ressenti parallèle d'une altérité 
chrétienne, elle-même profonde. Altérité qui voit se forger l'image positive de l'adversaire au détriment d'une dévalorisation radicale des alliés, quand bien même le Turc resterait le barbare désigné. Introduite par la pensée subjective de l'auteur, l'étude ne renonce pas à une analyse globale du conflit et des enjeux diplomatiques de la guerre à laquelle les éditeurs consacrent une synthèse des travaux parmi les plus fouillés sur le sujet, et singulièrement issus de l'historiographie turque. C'est l'un des fruits de cette heureuse collaboration que de transgresser les barrières de la langue pour convoquer à dessein la quintessence des études historiographiques internationales - même si l'on peut regretter que les atouts linguistiques de Ö. Bardakçi n'aient pas donné lieu à la retranscription de manuscrits orientaux, placés en vis-à-vis des extraits des principaux mémoires occidentaux utilisés au cours de l'étude et rassemblés, en partie, en fin de volume (chapitre 6). Rien ne semble oublié cependant pour favoriser l'approche du manuscrit et entrer de plain-pied dans la guerre : au-delà des multiples gravures, cartes, croquis, et de l'élaboration d'utiles glossaire et index qui facilitent la compréhension des enjeux et des termes purement techniques de la guerre, les auteurs semblent avoir réquisitionné, pour l'analyse du Mémoire, tous les témoignages disponibles utiles au portrait du capitaine Domenisse et à l'étude des interventions françaises au Levant.

3 Habilement construit, l'ouvrage se compose de six chapitres répondant de l'intérêt multiple du Mémoire dont les auteurs mettent en valeur chacune des facettes en un style ramassé et concis. Le premier tente de dresser le portrait de Domenisse, officier calviniste dont l'étude prend la mesure de l'héritage réformé et d'une vie passée dans les armées du roi, tout en faisant état de l'aspect initiatique du voyage d'Orient et de l'aura de la guerre contre l'Infidèle. L'étude resitue ensuite le propos de l'auteur, consacré à la fin de la guerre de Candie, dans le temps médian du troisième siège turc (1667-1669) et dans le temps long du conflit vénéto-ottoman (1645-1669). Le tout au profit d'une analyse de l'importance stratégique de la cité - clé de l'Empire vénitien dans la mer Égée - et des principaux enjeux politiques de la guerre, amorcée par Murad IV, poursuivie par Ibrahim I ${ }^{\mathrm{er}}$ et remportée, sur le terrain, par Fazil Ahmed Pacha, venu mettre fin à plus de quatre siècles de domination vénitienne. Ce second chapitre est aussi l'occasion de présenter l'état des forces des belligérants, et singulièrement des Français partis durant trois mois, de juin à août 1669, au secours des Italiens. Domenisse est l'un de ces six mille hommes dépêchés par Louis XIV pour répondre du dernier espoir de Venise et de la papauté de sauvegarder la place. Ce volet propose encore, par le biais des témoignages du siège de Candie, de revisiter les mythes et les réalités de la "guerre turque » et les aléas d'une lutte radicale qui, idéalisée dans la plupart des mémoires du temps, apparaît ici sous sa face sombre (dont témoignent le recours pendulaire à une violence sauvage, la lassitude et l'épuisement des troupes, la fréquente épouvante et terreur panique des soldats, la désorganisation, les problèmes de ravitaillement et les mutineries, mais aussi les transfuges dans le camp adverse et la mésintelligence chronique du commandement militaire franco-italien). Le troisième chapitre offre ensuite un panorama des expéditions françaises dans la guerre contre les Turcs, engagées respectivement en 1646, 1660, 1664 et 1669, en dépit de l'alliance fraternelle scellée entre le royaume et l'Empire Ottoman. Sans exclure les motivations d'ordre spirituel, les auteurs soulignent ici combien l'ingérence louis-quatorzienne dans le conflit semble procéder moins d'un esprit de croisade que des impératifs conjoncturels de la scène européenne (p.63), le souverain ne ralliant de surcroit le combat qu'après de longues négociations du Saint-Siège et n'acceptant de lutter que 
sous l'étendard pontifical, afin d'éviter la rupture ouverte avec le Sultan (p.69). L'analyse, fort attentive au jeu des intérêts respectifs, permet alors d'introduire de nombreux bémols à l'empreinte chrétienne du combat, et de relever une profonde inflexion de la lutte, désormais distante des engagements inscrits dans le sillage des anciens ligueurs, partis au début du siècle combattre les musulmans en Hongrie. Une réflexion fine qui, tout en rompant avec l'image idéalisée du conflit livrée par les récits passionnés des contemporains, continue d'interroger la forme néanmoins insolite de cette guerre aux accents d'ultime croisade qui compte aussi ses dévots et ses figures de martyrs (p. 67). Un dernier chapitre, précédant la retranscription annotée du Mémoire au chapitre $\mathrm{V}$, dissèque plus systématiquement le témoignage du capitaine qui, tout empreint d'une "culture de la confrontation» adossée aux représentations traditionnelles de l'Orient, n'en livre pas moins une vision ambiguë de l'ennemi musulman, partagée entre horreur et admiration (p. 79-93).

La richesse du manuscrit Domenisse ne réside pas dans la seule saveur d'une langue traversée d'occitanismes et émaillée de convictions abruptes. Si la syntaxe importe dans l'analyse conjointe de la perception de la guerre de Candie et dans la représentation de l'autre - qui ne se limite pas à l'adversaire ottoman, mais s'énonce aussi envers l'autorité pontificale et l'allié vénitien -, le récit s'impose pour ce qu'il laisse échapper du regard d'un officier subalterne en rupture avec les codes littéraires du genre et, plus encore, de l'empreinte protestante de l'auteur et de ce que F. Pugnière nomme les cultures de la coexistence, faisant ici cohabiter croyance individuelle, loyauté monarchique et sentiment chrétien d'un calviniste parti volontairement au Levant combattre pour « l'honneur du nom français » sous les bannières enchevêtrées du Très Chrétien, de Sa Sainteté Clément IX et de Saint-Marc. Féconde, la notion interroge aussi en amont l'importance des liens de sociabilité en la figure d'un protestant convaincu qui, n'ayant abjuré qu'en dernière instance, se trouvait pourtant bien noté de l'intendant Basville et apprécié d'un clergé alésien activiste en matière de contrainte religieuse envers ses propres coreligionnaires (p. 28). Parcours qui n'est pas sans témoigner d'une fierté contraignante, venue troubler les interprétations historiques trop manichéennes à l'égard des positions confessionnelles.

5 L'analyse générale est d'autant plus riche qu'elle ne se laisse pas subjuguer - en dépit de son titre - par l'emprise du mythe de la croisade et l'engagement à tonalité spirituelle, motivation amplement minorée par les études de Géraud Poumarède dont les auteurs s'emparent à bon escient dans leur interprétation très nuancée du phénomène. À partir de ce texte au demeurant mineur, $O$. Bardakçi et $F$. Pugnière jouent au contraire de l'éloquence d'une pensée complexe pour revisiter la notion d'un combat juste animé par un faisceau d'aspirations mêlant idéal nobiliaire, fidélité indéfectible au souverain et contribution à la gloire du royaume, implication dans le secours des princes chrétiens et espoir d'anoblissement.

6 L'étude du Mémoire fournit aussi l'occasion de revenir sur l'efficience du soutien français - longtemps déprécié -, d'éclairer les stratégies guerrières, les tensions et dissensions des cercles diplomatiques, et d'analyser la déclinaison des altérités culturelles et religieuses, turques et européennes. C'est dire le bénéfice de cette enquête croisée, ouverte à la fois à l'histoire militaire, sociale, religieuse, à l'histoire de la «psychologie collective» (Robert Sauzet) et du sensible qui s'intéresse aussi aux bruits de la guerre. L'historien des religions, des représentations et des idées y puisera de beaux exemples de la présence et de la convocation symétrique de Dieu (Mémoire: 
fol. $\left.3,8 \mathrm{v}^{\circ}, 9,14 \mathrm{v}^{\circ}, 16 \mathrm{v}^{\circ}, 21 \mathrm{v}^{\circ}, 22 \mathrm{v}^{\circ}\right)$, de l'aspect hideux de la guerre vécue, et des visions dépréciatives de Rome et des Italiens. Si Domenisse écrit en qualité d'officier des armées du roi, le mémoire n'est pas exempt d'une ironie toute calviniste raillant volontiers la couardise du neveu du pape, et jouant du sarcasme pour dénoncer l'inanité de la piété romaine qui, se répandant en distribution de médailles, chapelets et bénédictions papales, se trouvait peu à même d'effrayer l'ennemi turc (p.34). Récriminations auxquelles s'ajoutent celles, fort cinglantes, réservées aux Vénitiens, jugés versatiles, "fourbes en diable ", débauchés à souhait, et appelés dans leur cité de Candie - " asile de tous les scélérats de l'Europe ", en filigrane, comparable à l'impure Babylone et à Gomorrhe - à être justement châtiés par Dieu de leurs crimes et de leurs vices (p. 82). Le fait serait peut-être anecdotique s'il ne se révélait en contrepoint d'une reconnaissance appuyée de la ténacité, de la bravoure et de la loyauté de l'adversaire turc, combattant vaillamment au nom de son Dieu pour le triomphe de l'Islam et qui, se démarquant des poncifs, bouscule la vision traditionnellement admise du Barbare (p. 83-93). Témoignage fort des représentations du temps, le Mémoire Domenisse s'en distingue ainsi à plusieurs titres, et c'est bien tout l'attrait de cet ouvrage que d'en signaler les écarts éloquents malgré l'aversion d'alors pour les Mahométans.

Un mot encore de la teinte religieuse qui imprègne la presque totalité du manuscrit, bien au-delà des exemples déjà évoqués : ici dans les pavillons rouges des vaisseaux arborant saint Pierre et saint Paul $\left(\right.$ fol. $\left.3 \mathrm{v}^{\circ}\right)$ et dans les promesses des officiers faites à Dieu (fol. $\left.8 \mathrm{v}^{\circ}-9\right)$, là dans les exultations des religieux (fol. $\left.5 \mathrm{v}^{\circ}\right)$ et les cris d'assaut lancés au nom d'Allah (fol. $21 \mathrm{v}^{\circ}$ ), ailleurs dans les salves de flèches visant le camp chrétien pour saluer l'arrivée ou la mémoire d'un défunt bacha (fol. $22 \mathrm{v}^{\circ}$ ), plus indirectement peut-être dans les baisers solennels sur la barbe, pareils au serment du chrétien sur l'Évangile (fol. 23). Pour cela, comme pour sa rigueur scientifique et le partage d'un récit souvent émouvant face au vécu d'une expédition malheureuse et au spectacle d'une place chrétienne dévastée - «bouleversée » écrit Domenisse -, l'entreprise de Özkan Bardakçi et de François Pugnière s'avère, ainsi que le soulignait Robert Sauzet dans ses propos introductifs, une « incontestable réussite ».

\section{AUTEURS}

\section{CHRYSTEL BERNAT}

École Pratique des Hautes Études. 